\title{
Use of electronic apex locators may improve determination of working length
}

\author{
Abstracted from \\ Martins JN, Marques D, Mata A, Caramês J. \\ Clinical efficacy of electronic apex locators: systematic review. J Endod 2014; 40: 759-777. \\ Address for correspondence: Dr Jorge N. R. Martins, Instituto de Implantologia of Lisbon, \\ Av. Columbano Bordalo Pinheiro, 50 - 5 e 6, 1070-064 Lisboa, Portugal. E-mail: jnr_martins@yahoo.com.br
}

\section{Question: Are electronic apex locators (EALs) accurate?}

Data sources PubMed, LILACS, Science Direct, Cochrane Collaboration, NHS Evidence databases and in Evidence Based Dentistry and Journal of Evidence-Based Dental Practice. Study selection Randomised controlled trials (RCTs), clinical trials (CTs), diagnostic comparative studies and evaluation studies undertaken in adults where working length determination by both electronic and radiographic methods were used were considered. Data extraction and synthesis Two reviewers independently assessed study quality.

Results Twenty-one studies were included, five RCTs and 16 in vivo diagnostic test studies. There was considerable heterogeneity between the EALs used and the study designs and a narrative summary of the findings were presented. Working length measurement was compared using three different methods: distance to the radiographic apex in teeth undergoing root canal treatment, (11 studies); concordance between the comparative measurements with EAL and radiography, (two studies); distance to specific anatomic apical reference points evaluated after tooth extraction (four studies)ded. The body of evidence was once again assessed as of low quality.

Conclusions Within the limitations of this review, it is suggested that working length determination by using EAL may perform better than radiography alone.

\section{Commentary}

The ultimate goal of root canal treatment is to maintain the natural dentition by eliminating the root canal system as a source of infection that will lead to inflammation of the periodontium. Accurate determination of the apical extent of cleaning and shaping and ultimately obturation may interfere with treatment prognosis. ${ }^{1}$ The cementodentinal junction (CDJ) is the anatomical and histological landmark where the periodontal ligament begins and the pulp ends. Root canal preparation techniques aim to make use of this potential natural barrier between the contents of the canal and the apical tissues. ${ }^{2}$ Working length (WL) determination during root canal therapy has been greatly aided by electronic apex locators (EALs). They can save time when determining the working length, reduce patient exposure to radiation, and can be particularly helpful when the periapex is unclear on radiographs.

Although some early versions of EALs had issues with reliability and accuracy, the technology used to manufacture them has evolved over the years and the newer EALs can predict the location of the CDJ with a higher confidence level. ${ }^{2}$

This systematic review looked at the efficacy of EALs when compared to radiographs. The authors followed strict inclusion criteria. Randomised controlled trials and in vivo diagnostic test studies, that used third generation or higher EAL devices were reviewed. The authors noted that there was a lot of variability in the study methodologies, such as different predefined WL on a radiograph, ideal WL set at varied limits and different devices used, and commented on the need for standardised methodologies with the purpose of outcome comparison. The majority of studies had small sample sizes, which makes the results less reliable.

The conclusions drawn by the authors were based on a thorough assessment of the best evidence provided by the reviewed literature. They concluded that there is a high agreement rate between radiographs and EALs. When the two methods were compared, it is suggested that working length determination by using EAL may perform better than radiography alone.

Practice point

- EALs are important co-adjuvants in contemporary endodontic practice. The combination of radiographs and EALs can predictably measure the $\mathrm{WL}$ while exposing the patient to less radiation.

\section{Isabel Mello}

Department of Dental Clinical Sciences, Faculty of Dentistry, Dalhousie University, Halifax, Canada

1. Schaeffer MA, White RR, Walton RA. Determining the optimal obturation length: a meta-analysis of literature. J Endod 2005; 31: 271-274.

2. Gordon MP, Chandler NP. Electronic apex locators. Int Endod J 2004; 37: 425-437.

Evidence-Based Dentistry (2014) 15, 120. doi:10.1038/sj.ebd.6401066 